it may not need an animal use protocol or, if it turns out that it does need IACUC oversight, to walk the RDL through the steps it should follow to meet federal requirements. This is a wonderful teaching moment and opportunity to learn what each entity is doing, as well as to be a good neighbor. Furthermore, such contacts and exchange could open new avenues for collaborative research. Certainly, Covelli should seek more clarification from the RDL as to why it is seeking assistance from Great Eastern rather than setting up its own IACUC. Great Eastern University and the RDL need to start communicating effectively with each other, as either can have a positive or negative impact on the other's programs, depending on how they interact.

1. Institute for Laboratory Animal Research. Guide for the Care and Use of Laboratory Animals (National Academies Press, Washington, DC, 1996).

Owiny is University Veterinarian at Colorado State University, Fort Collins, CO. The views expressed in this article are his own and not those of Colorado State University.

\section{A word from USDA}

In response to the issues raised in this scenario, the United States Department of Agriculture, Animal and Plant Health Inspection Service, Animal Care (USDA/APHIS/AC) offers the following clarification and guidance:

There appears to be some confusion regarding the types of activities regulated by USDA. In general, most state health departments provide a clinical service, using animal blood and tissue samples, for their patients (the public) and are not required to be registered. However, diagnostic laboratories are required to comply with the Animal Welfare Act if they use live animals for the purpose of research. Testing is considered to be a component of "research and experimentation," and covered animals obtained specifically as test subjects may only be utilized under an IACUC-approved protocol.

In this scenario, it appears the bats were brought to the State Rabies Diagnostic Laboratory (SRDL) in order to obtain a clinical diagnosis. Euthanasia was provided as a service to those who brought in live animals (similar to what could be provided by a veterinary practitioner or animal pound). These activities alone would not require the SRDL to be registered as a research facility. If, however, the SRDL was soliciting the public for live bats specifically to collect research data (and not for clinical purposes), then it would need to be registered. The SRDL could be registered as a site under Great Eastern University's registration or as a separate entity.

\section{Chester Gipson, DVM}

Deputy Administrator USDA, APHIS, AC 